\title{
ORIGINAL ARTICLE \\ Sex differences in recent first-onset depression in an epidemiological sample of adolescents
}

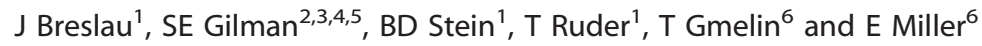

Prior studies provide limited and contradictory evidence regarding sex differences in the incidence of depression during adolescence, a critical period for development of the disorder. Data from six consecutive years (2009-2014) of a national survey of US adolescents aged $12-17(N=101685)$ are used to characterize sex differences in the incidence of depression by age and to compare recent first-onset and persistent depression with respect to impairment, suicide attempts, conduct problems and academic functioning. Projecting from age-specific incidence proportions, the cumulative incidence of depression between the ages of 12 and 17 is $13.6 \%$ among male and $36.1 \%$ among female subjects. The sex difference in incidence is significant at the age of 12 years $(5.2 \%$ in female versus $2.0 \%$ in male subjects, $P<0.0001)$, and it is significantly larger at ages of 13 through 17 years than at the age of 12 years $(P$-values $<0.05)$. Depression-related impairment is lower in recent first-onset than in persistent depression among female but not among male subjects. The prevalence of conduct problems and poor academic functioning is higher in both recent first-onset and persistent depression relative to those with no depression for both male and female subjects. The incidence of depression during adolescence is higher than that suggested by prior studies based on retrospective recall. Contrary to prior studies, evidence suggests that the sex difference in depression originates during childhood and grows in magnitude during adolescence. High levels of impairment, suicide attempts, conduct problems and poor academic functioning argue against a 'wait and see' approach to clinical treatment of recent first-onset depression.

Translational Psychiatry (2017) 7, e1139; doi:10.1038/tp.2017.105; published online 30 May 2017

\section{INTRODUCTION}

Major depression has a complex course characterized by intermittent acute episodes ${ }^{1}$ of varying length, ${ }^{2-4}$ severity $^{5}$ and frequency. ${ }^{6,7}$ An aim of epidemiological research has been to isolate and study specific temporal stages of the disorder to identify causal mechanisms that operate at different points in its course and to guide treatment efforts to the cases most likely to benefit from clinical care. ${ }^{8}$ The occurrence of depressive episodes during adolescence has received particularly close attention. It is during adolescence that risk for depression increases markedly ${ }^{9}$ and the functional impairments with the potential for long-term impact, such as failure to achieve academic milestones, begin to occur. ${ }^{10,11}$ However, prior studies provide limited information on the incidence of depression, that is, the first occurrence of a major depressive episode, during this age period. Information on incidence is important for testing etiologic hypotheses and for informing clinical work. For instance, there is evidence from some studies that male and female subjects have similar risk for depression during childhood, but female subjects begin to have higher risk when they reach their early teens. ${ }^{12-15}$ This evidence has led to studies of biological and social mechanisms for the apparent adolescent emergence of the sex difference in depression; ${ }^{16-18}$ however, the underlying pattern has not been rigorously tested in epidemiological data. From a clinical perspective, it may be the case that incident cases are associated with lower levels of impairment than persistent cases, supporting the suggestion that clinicians should 'wait and see'19 whether recent-onset cases become persistent before initiating treatment.

Several national studies have examined the prevalence of depression in adolescent samples, some using rating scales ${ }^{20,21}$ and some using diagnostic interviews. ${ }^{9,22}$ These studies are limited because prevalent cases include a mixture of persistent and recent-onset cases. ${ }^{23}$ Two methodological approaches have been taken to study incidence of the disorder separate from persistence. Longitudinal studies with assessments of depression at multiple time points during childhood and adolescence have found that higher prevalence among female subjects emerges in early adolescence; however, these studies have limited power to detect changes in sex differences across time. ${ }^{12,24}$ The one longitudinal study to examine sex differences in incident cases, a birth cohort study from New Zealand, reported that the incidence of depression among female subjects began to exceed that among males between the ages of 13 and 15 years. ${ }^{13}$ However, that study may not have detected a sex difference at earlier ages because of the small number of prevalent cases at the initial assessment at the age of 11 years.

In contrast, single-interview studies with larger sample sizes have enabled researchers to reconstruct incidence curves using retrospective reports of depression at the age of onset. ${ }^{9,25}$ These 'retrospective incidence' studies suggest that sex difference in

\footnotetext{
${ }^{1}$ Health Division, RAND Corporation, Pittsburgh, PA, USA; ${ }^{2}$ Division of Intramural Population Health Research, Health Behavior Branch, Eunice Kennedy Shriver National Institute of Child Health and Human Development, Bethesda, MD, USA; ${ }^{3}$ Department of Social and Behavioral Sciences, Harvard TH Chan School of Public Health, Boston, MA, USA; ${ }^{4}$ Department of Epidemiology, Harvard TH Chan School of Public Health, Boston, MA, USA; ${ }^{5}$ Department of Mental Health, Johns Hopkins Bloomberg School of Public Health, Baltimore, MD, USA and ${ }^{6}$ Division of Adolescent and Young Adult Medicine, School of Medicine, University of Pittsburgh, Pittsburgh, PA, USA . Correspondence: Dr J Breslau, Health Division, RAND Corporation, 4570 Fifth Avenue, Pittsburgh, PA 15213, USA.
}

E-mail: jbreslau@rand.org

Received 3 April 2017; accepted 10 April 2017 
depression emerges prior to adolescence. ${ }^{9,26}$ A major limitation of these studies is that they underestimate incidence due to recall bias with many cases going unreported because they are forgotten over time. ${ }^{27,28}$

In this study we use a large nationally representative sample of adolescents at the ages of 12-17 years, drawn from multiple years of a repeated cross-sectional survey, to address these limitations. We examine sex differences in the incidence of recent first-onset depression, defined by reports of first-in-lifetime depressive episode with onset within 1 year of age prior to interview. This approach mitigates the under-reporting bias that has been shown to affect retrospective incidence studies. ${ }^{29}$ The large sample size provides sufficient power to detect sex differences at each year of age, which has been lacking in longitudinal studies.

The study has two goals. First, we aim to compare sex-specific incidence of recent first-onset depression over this critical age range, during which, according to prior research, the excess risk of depression among female subjects emerges. This hypothesis has never been tested with data on recent first onset of depression in a large epidemiological sample. Second, we test whether recent first-onset cases differ from persistent cases, that is, prevalent cases with prior onset, with respect to key clinical and functional correlates of adolescent depression-impairment, suicide attempts, conduct problems and school functioning. ${ }^{30}$ We expect persistent cases, due to their duration, to be associated with more severe impairment, higher likelihood of suicide attempt, higher levels of conduct problem and poorer school functioning compared with recent first-onset cases. If this is the case, then persistence over time may be a clinically meaningful indicator for treatment needed among adolescents with depression.

\section{MATERIALS AND METHODS}

\section{Sample}

Data on adolescents aged 12-17 years from six consecutive years (20092014) of the National Survey of Drug Use and Health (NSDUH), an annual cross-sectional survey conducted in a representative sample of the US population, were analyzed. The NSDUH is based on a stratified multistage area probability sample designed to be representative of the entire US and of each of the 50 states and the District of Colombia. The NSDUH has an annual sample size target of 67500 respondents, with $25 \%$ falling in the 12-17 age group studied here. ${ }^{31}$ The weighted overall response rate for the complete sample (all ages, not just adolescents), which is the screening response rate multiplied by the interview response rate, ranges from 66.8 to $58.3 \%$ during this time period. The interview response rate is higher for the adolescent sample than that for the adult sample. Sample weights adjust for non-response and demographic characteristics at the household and individual levels as described in methodological reports available on the survey website (https://www.samhsa.gov/data/population-data-nsduh/ reports?tab $=39$ ).

Interviews were completed in-person by trained interviewers. Sensitive questions, including mental health assessments, were asked using computer-assisted methods, where respondents enter their responses directly into a secure computer. Questions may be read aloud by the interviewer, read by the respondent or played to the respondent through headphones. Computer-assisted methods help maintain privacy and confidentiality and reduce response bias. ${ }^{32}$ Procedures for analysis of the publicly accessible NSDUH data were approved by the RAND Human Subjects Protection Committee.

\footnotetext{
Measures

Depression. Depression was assessed according to Diagnostic and Statistical Manual of Mental Disorders, Version IV criteria using an instrument adapted to the A-CASI format from that used in the National Comorbidity Survey Replication-Adolescent Supplement. ${ }^{31}$ A clinical reappraisal study of the National Comorbidity Survey ReplicationAdolescent Supplement found high sensitivity and specificity for the instrument when depression assessments were compared with blinded clinical interviews. ${ }^{33}$ Respondents reporting a major depressive episode are asked their age at the first time that they had such an episode and whether
}

they had been experiencing such episodes within the 12 months prior to the interview. Information on timing was used to classify respondents who met criteria for a depressive episode into the following three mutually exclusive categories:

Recent first onset: Respondent reports age at first-in-lifetime depressive episode as either their current age or at 1 year prior to their current age. Persistent: Respondent reports being in a depressive episode within the past 12 months and onset of their first depressive episode at more than 1 year of age prior to their current age.

Remitted: Respondent reports not having been in an episode within the past 12 months and onset of their first depressive episode at more than 1 year of age prior to their current age.

Impairment. The Sheehan Disability Scale was used to assess depressionrelated impairment among respondents reporting a depressive episode in the past 12 months (that is, categories 1 and 2 above). The Sheehan Disability Scale uses a visual analog scale to assess limitations on a scale of 0-10 in each of four domains: chores at home, school or work, close relationships with family and social life. ${ }^{34,35} \mathrm{~A}$ rating of $\geqslant 7$ in at least one domain is considered to be severe impairment.

Suicide attempt. Respondents reporting depressive symptoms within the past year were asked whether they had attempted suicide during a depressive episode.

Conduct problems. Respondents were asked how frequently they had engaged in the following five conduct problems within the past year: (1) getting into a serious fight at school or work; (2) taking part in a fight in which one group fought against another; (3) carrying a handgun; (4) selling illegal drugs; and (5) stealing or trying to steal something worth more than $\$ 50$. Each conduct problem was coded as present if the respondent reported one or more occurrence during the past year.

Academic functioning. Respondents were asked a series of four Likert style questions regarding their school experiences within the past year. Questions addressed how often they felt that their school work is meaningful and important, how important they think their school work will be later in life, how interesting their courses are and how often teachers let them know they were doing a good job. Responses to these items were used to define indicators of poor academic functioning, for example, seldom or never versus sometimes or always. Respondents were also asked their average grades during their most recent school grading period. Responses were used to define an indicator of grade average of $\mathrm{C}$ or lower.

\section{Statistical analysis}

Incidence proportions, that is, the number of recent first-onset cases divided by the total number of individuals without a prior depressive episode (that is, remitted or persistent depression), were calculated separately for males and female subjects at the ages of 12-17 years. Age variation in the sex difference in incidence proportion was tested using a logistic regression model. Age-specific annual incidence rates were calculated by dividing the incidence proportions by 1.5 , as on average respondents reported on 18 months prior to the interview. Cumulative incidence was calculated by multiplying each age-specific rate by the proportion of the population without prior depression and summing across ages. Among respondents with past-year depression, recent firstonset cases were compared with persistent cases with respect to impairment and occurrence of suicide attempts. Associations of depression category with each correlate were estimated in logistic regression models with statistical control for age, sex and their interaction. Models with statistical interactions between sex and depression category had significantly better fit than models without these interactions as evidenced by lower Akaike information criteria ${ }^{36}$ and Bayesian information criteria. ${ }^{37}$ Consequently, all results are shown separately for female subjects and males.

Among the full sample, recent first-onset cases were compared with persistent cases and those reporting no depression with respect to conduct problems and indicators of poor school functioning (remitted cases were included in the no-depression category). Associations of the depression categories with each correlate were estimated in logistic regression models with statistical controls for age, sex and their 
Table 1. Prevalence of depression in adolescents ages 12-17 years by sex, NSDUH 2009-2014

\begin{tabular}{|c|c|c|c|}
\hline Depression category & Total sample $(\mathrm{n}=101685 ; \%)$ & Males $(\mathrm{n}=51$ 838; \%) & Females $(n=49847 ; \%)$ \\
\hline Recent first-onset depression & 6.5 & 3.5 & 9.6 \\
\hline Any depression & 14.0 & 8.0 & 20.2 \\
\hline
\end{tabular}

interaction. As with impairment results, because of statistical interactions between sex and depression categories, results are presented separately for female subjects and males.

All statistical analyses were conducted using STATA survey procedures to adjust s.e.'s for the survey design. ${ }^{38}$ The statistical programming code is available from the authors.

\section{RESULTS}

Prevalence of depression

The sample is $48.9 \%$ female and roughly equally distributed across ages, ranging from $15 \%$ to $17 \%$ across the six age groups. Of the entire sample, $14 \%$ report having had an episode of depression (Table 1). Recent first-onset depression is more common than persistent or remitted depression, accounting for just under half of all cases for both males and female subjects. Prevalence is higher among female subjects than males among all three categories.

Sex differences in depression incidence by age

The incidence proportion of recent first-onset depression is higher in female subjects than in males at all ages (Figure 1a). At the age of 12 , the incidence proportion is $5.7 \%$ among female subjects and $2.0 \%$ among males. The incidence proportion is highest at the age of 15 for female subjects, when it reaches $13.2 \%$, and at the age of 17 for males, when it reaches $4.9 \%$. The incidence difference between female subjects and males also varies across ages. The incidence difference at the age of 12 is $3.7 \%$, and it is significantly larger at each subsequent age, reaching $8.8 \%$ at the age of 15 before declining slightly to $5.7 \%$ at the age of $17(P$ values for pairwise comparisons all $<0.05)$. In relative terms, female subjects are 2.8 times more likely to develop depression than males at the age of 12 and between 3.1 and 4.0 times as likely as males to develop depression at the ages 13 through 16 . The relative risk of 2.2 at the age of 17 is lower than that at the age of 12 . On the basis of the age- and sex-specific incidence proportions, nearly three times as many female subjects (36.1\%) as males (13.6\%) will experience first onset of depression between the ages of 12 and 17 (Figure $1 \mathrm{~b}$ ).

\section{Impairment due to depression}

Table 2 shows the prevalence of moderate or severe impairment across four domains and suicide attempts among respondents with past-year depression for persistent and first-onset cases. Among female subjects, persistent cases have significantly higher levels of impairment for all domains and higher rates of suicide attempts than recent first-onset cases. In contrast, among males there is a significant difference between persistent and recent first-onset cases for only one of the four domains, chores at home, and, for that domain, the prevalence is higher among the recent first-onset cases. There is no significant difference among males in prevalence of suicide attempts.
Conduct problems and academic functioning

The top panel of Table 3 shows the prevalence of conduct problems in those with recent first-onset depression, persistent depression and no depression by sex. Conduct problems are significantly more common in the two groups with depression than in the no-depression group in all but 2 of the 26 possible comparisons; the prevalence of 'carried a handgun' does not differ between the persistent and no-depression groups among males $(P=0.0655)$ or between the recent first-onset and no-depression groups among female subjects $(P=0.6401)$. There are significant differences between the recent first-onset and persistent depression groups, but only among female subjects. Among female subjects the persistent depression group has significantly higher prevalence for four of the six conduct problems and for the indicator of having at least two of the conduct problems.

The bottom panel of Table 3 shows comparisons across the same groups for measures of poor academic functioning. The prevalence of poor academic functioning indicators is higher in two groups with depression than in the no-depression group in all but 1 of the 24 possible comparisons; the prevalence of 'Grades in the last semester $C$ or lower' does not differ between the persistent and no-depression groups for males $(P=0.0653)$. There are differences between recent first-onset and persistent cases with respect to two of the five indicators, but only among males, and these differences go in opposite directions for different indicators. Males with persistent depression are more likely than males with recent first-onset depression to have three or more indicators of poor academic functioning.

\section{DISCUSSION}

This study of the incidence of first onset of depression in a nationally representative sample of adolescents found projections of cumulative incidence of recent first-onset depression of $13.6 \%$ for males and $36.1 \%$ for female subjects between the ages of $12-$ 17 , substantially higher than estimates based on direct tabulation of recalled cases. For comparison, the lifetime prevalence of depression in the sample was $8 \%$ among males and $20.2 \%$ among female subjects. This finding confirms the suggestion that estimates of lifetime prevalence based on retrospective recall underestimate the cumulative prevalence due to under-reporting of prior depressive episodes. ${ }^{27}$ Our approach for examining first onset and cumulative incidence makes an important contribution, given that retrospective recall can be influenced by some clinical characteristics of the disorder, including persistence over time. ${ }^{28,39}$

Based primarily on longitudinal studies, which have found that the sex difference in prevalence of depression first emerges between the ages of 12 and 15, researchers have suggested that risk factors first appearing during adolescence must account for the high prevalence of the disorder among female subjects relative to males. ${ }^{40-42}$ Evidence from this study partially contradicts this view. The data suggest that the sex difference in incidence of depression is already quite large among the 12-yearold respondents, who were reporting onsets that occurred when 


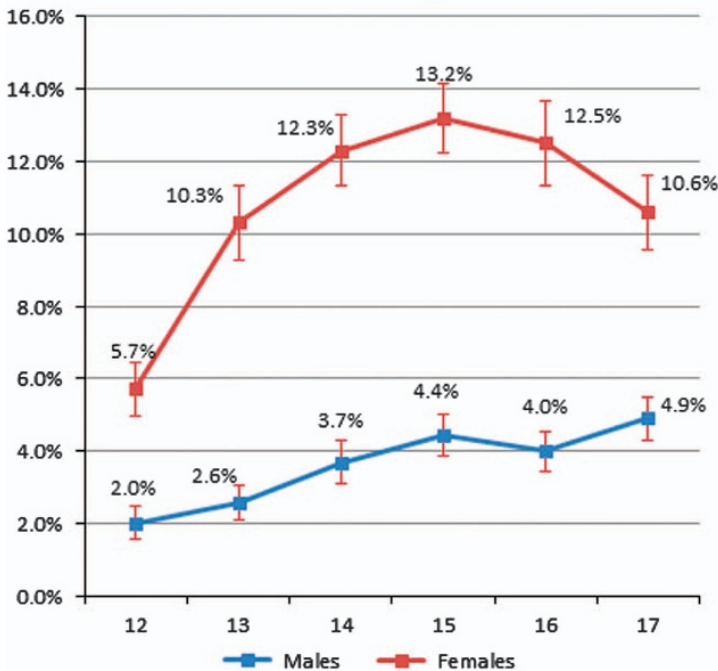

b Projected Cumulative Incidence

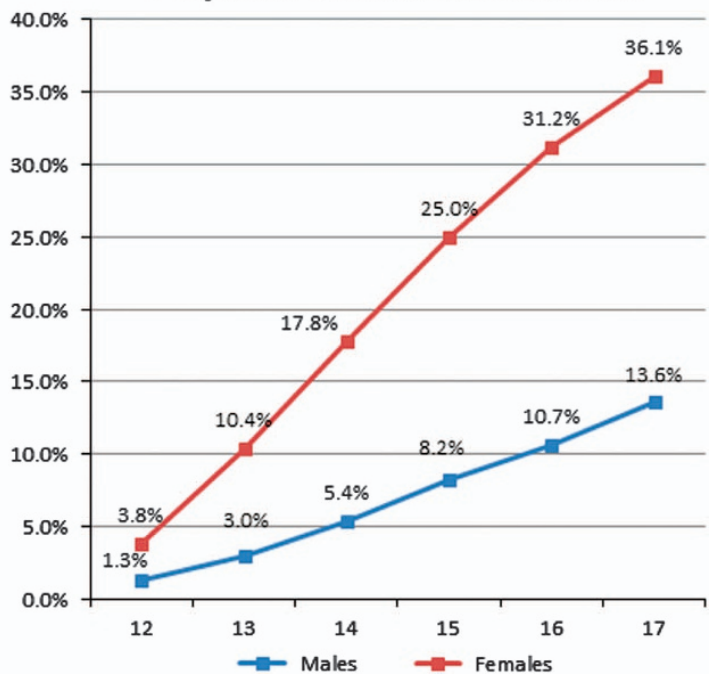

Figure 1. Occurrence of first-onset depression among adolescents, by age and sex.

\begin{tabular}{|c|c|c|c|c|c|c|}
\hline \multirow[t]{2}{*}{ Impairment domain } & \multicolumn{3}{|c|}{ Males } & \multicolumn{3}{|c|}{ Females } \\
\hline & Persistent (\%) & Recent first onset (\%) & P-value ${ }^{a}$ & Persistent (\%) & Recent first onset (\%) & P-value ${ }^{\mathrm{a}}$ \\
\hline Chores at home & 29.8 & 34.3 & 0.0405 & 34.0 & 29.1 & 0.0002 \\
\hline School or work & 35.7 & 36.9 & 0.4224 & 40.6 & 34.8 & 0.0012 \\
\hline Family relationships & 40.3 & 42.4 & 0.3177 & 56.9 & 49.8 & 0.0003 \\
\hline Social life & 43.6 & 44.5 & 0.3139 & 51.0 & 46.8 & 0.0158 \\
\hline Any domain & 68.3 & 68.7 & 0.4825 & 76.7 & 68.5 & $<0.0001$ \\
\hline Suicide attempt & 16.3 & 15.1 & 0.8208 & 28.7 & 15.8 & $<0.0001$ \\
\hline
\end{tabular}

${ }^{\mathrm{a}} P$-values correspond to design adjusted $X^{2}$-tests $(1$ degree of freedom) for equivalence of the proportions with impairment between the persistent and recent first-onset categories.

they were as young as 11 years for age. The finding that the sex difference in depression begins prior to adolescence, and thus may be influenced by factors occurring during childhood, is consistent with prior studies based on retrospective reports. ${ }^{9}$ There is also prior evidence that sex differences in childhood anxiety disorders have a role in sex differences in depression later in life. ${ }^{43}$ The hypothesis that risk factors appearing during adolescence contribute to the sex difference in depression is also partially supported by the finding that the difference between males and female subjects in incidence of depression increases with age.

We hypothesized that indicators of impairment, conduct problems and poor academic functioning, which are common correlates of depression among adolescents, would be more common among those with persistent depression than those with recent first-onset depression. The results support this hypothesis, but only for female subjects. Among respondents with past-year depression, we found substantially higher levels of impairment and attempted suicide among female subjects with persistent depression compared with recent-onset depression, but no comparable pattern among males. This pattern is perhaps most striking for suicide attempts; among males there is no difference across the groups (16.3\% versus $15.1 \%$ ), whereas among female subjects those with recent first onset are nearly half as likely to have attempted suicide than those with persistent depression
(15.8\% versus $28.7 \%$ ). Notably, the National Comorbidity Survey Replication-Adolescent Supplement found a threefold difference in the prevalence of suicide attempt between those with severe versus mild or moderate depression. ${ }^{9}$ Sex differences in this relationship deserve additional research attention.

The findings regarding conduct problems are similar; the prevalence of conduct problems is higher among the persistent than among the recent first-onset group among female subjects but not among males. With respect to indicators of poor academic functioning, differences between recent first-onset and persistent depression groups were generally not statistically significant, although there was some evidence of poorer functioning in the persistent group among males.

The analyses presented here do not address the direction of causality between depression, impairment, conduct problems and poor academic functioning. Rather, the clinical motivation for testing the difference between recent first-onset and persistent cases with respect to these correlates of depression was to examine whether it might be justifiable to prioritize persistent cases, treating recent first-onset cases with a 'wait and see' approach, which research suggests is common among clinicians. ${ }^{19}$ Even for those correlates where the hypothesis was supported by the evidence, that is, stronger associations were found for persistent than for recent first-onset cases, this clinical implication was not supported by the data. Differences between persistent 
Table 3. Comparison of externalizing behaviors and academic function between recent-onset depression, persistent depression and no past-year depression groups for males and female subjects ${ }^{a}$

\begin{tabular}{|c|c|c|c|c|c|c|}
\hline & \multicolumn{3}{|c|}{ Males } & \multicolumn{3}{|c|}{ Females } \\
\hline & $\begin{array}{l}\text { Recent onset } \\
\text { (\%) }\end{array}$ & $\begin{array}{l}\text { Persistent } \\
\text { (\%) }\end{array}$ & $\begin{array}{c}\text { No depression } \\
\text { (\%) }\end{array}$ & $\begin{array}{l}\text { Recent onset } \\
\text { (\%) }\end{array}$ & $\begin{array}{l}\text { Persistent } \\
\text { (\%) }\end{array}$ & $\begin{array}{c}\text { No depression } \\
\text { (\%) }\end{array}$ \\
\hline \multicolumn{7}{|l|}{ Conduct problems } \\
\hline Gotten into a serious fight at school/work & 33.2 & 31.1 & 21.4 & 25.9 & 26.0 & 13.7 \\
\hline Taken part in fight where group fought another group & 21.2 & 18.5 & 12.7 & 16.7 & 15.9 & 9.9 \\
\hline Attacked someone w/intent to seriously hurt them & 16.9 & 18.4 & 6.4 & 9.6 & 12.0 & 3.7 \\
\hline Two or more conduct problems & 25.8 & 25.5 & 12.4 & 15.3 & 17.2 & 6.8 \\
\hline \multicolumn{7}{|l|}{ Academic functioning } \\
\hline School work meaningful= seldom, never & 35.8 & 41.6 & 19.6 & 30.8 & 33.4 & 16.2 \\
\hline Importance of learning = somewhat/very unimportant & 23.0 & 28.2 & 12.3 & 21.1 & 24.5 & 11.0 \\
\hline \multicolumn{7}{|c|}{$\begin{array}{l}\text { a Statistical tests were conducted using logistic regression models with } P=0.05 \text {. Proportions are significantly higher in the recent first-onset and persistent } \\
\text { categories than in the no-depression category for all indicators with the following three exceptions: ( } 1 \text { ) carried a handgun does not differ between recent first- } \\
\text { onset and no-depression groups among female subjects }(P=0.6401) \text {; ( } 2 \text { carried a handgun does not differ between persistent and no-depression groups for } \\
\text { males }(P=0.0655) \text {; and (3) grades in the last semester } C \text { or lower does not differ between the persistent and no-depression groups for males }(P=0.0653) \text {. } \\
\text { Figures in bold indicate statistically significant differences between the recent-onset and persistent groups. }\end{array}$} \\
\hline
\end{tabular}

and recent first-onset cases are small in comparison with the differences between both groups and those with no depression. For instance, the prevalence of suicide attempt in the past year was over $15 \%$ for both female subjects and males with recent firstonset depression. This evidence clearly does not support taking a 'wait and see' approach to recent first-onset cases of depression in clinical settings.

These findings should be interpreted in light of two limitations. First, although the method of estimating the incidence proportion reduces the impact of recall bias, as those with recent disorders are more likely to report accurately, it is not completely free from this bias, as it still relies on retrospective report of the occurrence (or non-occurrence) and age of onset of depressive episodes. Specifically, some respondents with prior episodes of depression will have forgotten those episodes and be incorrectly classified as having never had depression. As the denominators for the incidence proportions will therefore be inflated, the estimated incidence proportions will be biased downward. However, this downward bias is likely to be smaller than the more pervasive impact of recall bias on reporting of lifetime prevalence. This means that our projections of the cumulative incidence between the ages of 12 and 17 are likely to be underestimates of the true cumulative incidence during this age period.

Second, the assessments of impairment, conduct problems and academic functioning are based on self-report. Owing to the influence of depression on self-perception, it is likely that these assessments are biased toward higher prevalence among those with depression. This bias is unlikely to influence comparisons between recent first-onset cases and prior onset past-year cases. Third, the persistent depression category in this study combines cases that are chronic with cases that are recurrent, although research suggests there are clinically significant differences between these subgroups. ${ }^{44,45}$

The high prevalence and varied course of depression makes it challenging to study in epidemiologic samples. However, due to the low level of clinical treatment, ${ }^{46}$ these studies are essential to understand its causes and its individual and societal burdens. The strategy used here to compile a sample of first-onset cases helps advance our understanding of the basic epidemiology of the disorder and may contribute to a new strategy for etiologic studies.

\section{CONFLICT OF INTEREST}

The authors declare no conflict of interest.

\section{ACKNOWLEDGMENTS}

This research was supported by a grant from the National Institute of Minority Health and Health Disparities (R01MD010274) and by the Intramural Research Program of the Eunice Kennedy Shriver National Institute of Child Health and Human Development.

\section{REFERENCES}

1 Kovacs M, Obrosky S, George C. The course of major depressive disorder from childhood to young adulthood: recovery and recurrence in a longitudinal observational study. J Affect Disord 2016; 203: 374-381.

2 Spijker J, de Graaf R, Bijl RV, Beekman AT, Ormel J, Nolen WA. Duration of major depressive episodes in the general population: results from The Netherlands Mental Health Survey and Incidence Study (NEMESIS). Br J Psychiatry 2002; 181: 208-213.

3 Spijker J, de Graaf R, Bijl RV, Beekman AT, Ormel J, Nolen WA. Determinants of persistence of major depressive episodes in the general population. Results from the Netherlands Mental Health Survey and Incidence Study (NEMESIS). J Affect Disord 2004; 81: 231-240.

4 Eaton WW, Shao H, Nestadt G, Lee BH, Bienvenu OJ, Zandi P. Population-based study of first onset and chronicity in major depressive disorder. Arch Gen Psychiatry 2008; 65: 513-520.

5 Kruijshaar ME, Hoeymans N, Bijl RV, Spijker J, Essink-Bot ML. Levels of disability in Major depression - findings from the Netherlands Mental Health Survey and Incidence Study (NEMESIS). J Affect Disord 2003; 77: 53-64.

6 Hardeveld F, Spijker J, De Graaf R, Nolen WA, Beekman AT. Recurrence of major depressive disorder and its predictors in the general population: results from the Netherlands Mental Health Survey and Incidence Study (NEMESIS). Psychol Med 2013; 43: 39-48. 
7 Hardeveld F, Spijker J, De Graaf R, Nolen WA, Beekman AT. Prevalence and predictors of recurrence of major depressive disorder in the adult population. Acta Psychiatr Scand 2010; 122: 184-191.

8 Monroe SM, Harkness KL. Recurrence in major depression: a conceptual analysis. Psychol Rev 2011; 118: 655-674.

9 Avenevoli S, Swendsen J, He JP, Burstein M, Merikangas KR. Major depression in the national comorbidity survey-adolescent supplement: prevalence, correlates, and treatment. J Am Acad Child Adolesc Psychiatry 2015; 54: 37-44 e2.

10 Copeland WE, Miller-Johnson S, Keeler G, Angold A, Costello EJ. Childhood psychiatric disorders and young adult crime: a prospective, population-based study. Am J Psychiatry 2007; 164: 1668-1675.

11 Breslau J, Miller E, Chung WJJ, Schweitzer JB. Childhood and adolescent onset psychiatric disorders, substance use, and failure to graduate high school on time. J Psychiatr Res 2011; 45: 295-301.

12 Angold A, Costello EJ, Erkanli A, Worthman CM. Pubertal changes in hormone levels and depression in girls. Psychol Med 1999; 29: 1043-1053.

13 Hankin BL, Abramson LY, Moffitt TE, Silva PA, McGee R, Angell KE. Development of depression from preadolescence to young adulthood: emerging gender differences in a 10-year longitudinal study. J Abnorm Psychol 1998; 107: 128-140.

14 Cohen P, Cohen J, Kasen S, Velez CN, Hartmark C, Johnson J et al. An epidemiological study of disorders in late childhood and adolescence--l. Age- and gender-specific prevalence. J Child Psychol Psychiatry 1993; 34: 851-867.

15 Thapar A, Collishaw S, Pine DS, Thapar AK. Depression in adolescence. Lancet 2012; 379: 1056-1067.

16 Hankin BL. Development of sex differences in depressive and co-occurring anxious symptoms during adolescence: Descriptive trajectories and potential explanations in a multi-wave prospective study. J Clin Child Adolesc Psychol 2009; 38: 460-472.

17 Cyranowski JM, Frank E, Young E, Shear MK. Adolescent onset of the gender difference in lifetime rates of major depression: a theoretical model. Arch Gen Psychiatry 2000; 57: 21-27.

18 Naninck EF, Lucassen PJ, Bakker J. Sex differences in adolescent depression: do sex hormones determine vulnerability? J Neuroendocrinol 2011; 23: 383-392.

19 Schumann I, Schneider A, Kantert C, Lowe B, Linde K. Physicians' attitudes, diagnostic process and barriers regarding depression diagnosis in primary care: a systematic review of qualitative studies. Fam Pract 2012; 29: 255-263.

20 Rushton JL, Forcier M, Schectman RM. Epidemiology of depressive symptoms in the National Longitudinal Study of Adolescent Health. J Am Acad Child Adolesc Psychiatry 2002; 41: 199-205.

21 Maslowsky J, Schulenberg JE, Zucker RA. Influence of conduct problems and depressive symptomatology on adolescent substance use: developmentally proximal versus distal effects. Dev Psychol 2014; 50: 1179-1189.

22 Mojtabai R, Olfson M, Han B. National trends in the prevalence and treatment of depression in adolescents and young adults. Pediatrics 2016; 138: e20161878.

23 Klein DN, Glenn CR, Kosty DB, Seeley JR, Rohde P, Lewinsohn PM. Predictors of first lifetime onset of major depressive disorder in young adulthood. $J$ Abnorm Psychol 2013; 122: 1-6.

24 Hankin BL, Young JF, Abela JR, Smolen A, Jenness JL, Gulley LD et al. Depression from childhood into late adolescence: Influence of gender, development, genetic susceptibility, and peer stress. J Abnorm Psychol 2015; 124: 803-816.

25 Kessler RC, Berglund P, Demler O, Jin R, Merikangas KR, Walters EE. Lifetime prevalence and age-of-onset distributions of DSM-IV disorders in the National Comorbidity Survey Replication. Arch Gen Psychiatry 2005; 62: 593-602.

26 Rohde P, Lewinsohn PM, Klein DN, Seeley JR, Gau JM. Key characteristics of major depressive disorder occurring in childhood, adolescence, emerging adulthood, adulthood. Clin Psychol Sci 2013; 1: 41-53.

27 Moffitt TE, Caspi A, Taylor A, Kokaua J, Milne BJ, Polanczyk G et al. How common are common mental disorders? Evidence that lifetime prevalence rates are doubled by prospective versus retrospective ascertainment. Psychol Med 2010; 40: 899-909.
28 Simon GE, VonKorff M. Recall of psychiatric history in cross-sectional surveys: implications for epidemiologic research. Epidemiol Rev 1995; 17: 221-227.

29 Wells JE, Horwood LJ. How accurate is recall of key symptoms of depression? A comparison of recall and longitudinal reports. Psychol Med 2004; 34: 1001-1011.

30 Wilson S, Vaidyanathan U, Miller MB, McGue M, lacono WG. Premorbid risk factors for major depressive disorder: are they associated with early onset and recurrent course? Dev Psychopathol 2014; 26(4 Pt 2): 1477-1493.

31 Center for Behavioral Health Statistics and Quality. 2014 National Survey on Drug Use and Health: Methodological summary and definitions. Substance Abuse and Mental Health Services Administration: Rockville, MD, USA, 2015.

32 Gfroerer J, Eyerman J, Chromy JR. Redesigning an Ongoing National Household Survey: Methodological issues. Substance Abuse and Mental Health Services Administration, Office of Applied Studies: Rockville, MD, USA, 2002, Contract No.: HHS Publication No. SMA 03-3768.

33 Kessler RC, Avenevoli S, Green JG, Gruber MJ, Guyer M, He Y et al. The National Comorbidity Survey Adolescent Supplement (NCS-A): III. Concordance of DSM-IV/ CIDI diagnoses with clinical reassessments. J Am Acad Child Adolesc Psychiatry 2009; 48: 386-399.

34 Leon AC, Olfson M, Portera L, Farber L, Sheehan DV. Assessing psychiatric impairment in primary care with the Sheehan Disability Scale. Int J Psychiatry Med 1997; 27: 93-105.

35 Sheehan DV. Establishing the real cost of depression. ManagCare 2002; 11(8 Suppl): 7-10.

36 Akaike H. Factor-analysis and AIC. Psychometrika 1987; 52: 317-332.

37 Schwarz G. Estimating the dimension of a model. Ann Statist 1978; 6: 461-464.

38 StataCorp.. Stata Statistical Software, Release 13. StataCorp LP: College Station, TX, USA, 2015.

39 Costello EJ, Copeland W, Angold A. Trends in psychopathology across the adolescent years: what changes when children become adolescents, and when adolescents become adults? J Child Psychol Psychiatry 2011; 52: 1015-1025.

40 Hyde JS, Mezulis AH, Abramson LY. The ABCs of depression: Integrating affective, biological, and cognitive models to explain the emergence of the gender difference in depression. Psychol Rev 2008; 115: 291-313.

41 Nolen-Hoeksema S. Gender differences in depression. Curr Dir Psychol 2001; 10: 173-176.

42 Hankin BL, Abramson LY. Development of gender differences in depression: an elaborated cognitive vulnerability-transactional stress theory. Psychol Bull 2001; 127: 773-796.

43 Breslau N, Schultz L, Peterson E. Sex differences in depression: a role for preexisting anxiety. Psychiatry Res 1995; 58: 1-12.

44 Hölzel L, Härter M, Reese C, Kriston L. Risk factors for chronic depression - a systematic review. J Affect Disord 2011; 129: 1-13.

45 Klein DN, Santiago NJ. Dysthymia and chronic depression: introduction, classification, risk factors, and course. J Clin Psychol 2003; 59: 807-816.

46 Merikangas KR, He J-p, Burstein M, Swendsen J, Avenevoli S, Case B et al. Service utilization for lifetime mental disorders in U.S. adolescents: results of the National Comorbidity Survey-Adolescent Supplement (NCS-A). J Am Acad Child Adolesc Psychiatry 2011; 50: 32-45.

This work is licensed under a Creative Commons AttributionNonCommercial-ShareAlike 4.0 International License. The images or ther third party material in this article are included in the article's Creative Commons license, unless indicated otherwise in the credit line; if the material is not included under the Creative Commons license, users will need to obtain permission from the license holder to reproduce the material. To view a copy of this license, visit http:// creativecommons.org/licenses/by-nc-sa/4.0/

(c) The Author(s) 2017 